\title{
Reconfigurable OFDM based transceiver for Wireless Communication
}

\author{
${ }^{1}$ Jayashree Chavan, ${ }^{2}$ Prof. Sadhana C, ${ }^{3}$ Prof. Basavaling Hiremath \\ ${ }^{1}$ M.Tech, VLSI Design \& Embedded System, LAEC, Bidar \\ ${ }^{2}$ Assistant Professor, Dept. of E\&CE, LAEC, Bidar \\ ${ }^{3}$ Assistant Professor, Dept. of E\&CE, LAEC, Bidar
}

\begin{abstract}
Orthogonal Frequency Division Multiplexing (OFDM) is a multi-carrier modulation technique which is very much popular in new wireless networks of IEEE standard. It has improved the quality of longdistance communication by eliminating Inter Symbol Interference (ISI) and improving Signal-to-Noise Ratio (SNR) And Multipath Fading. Reconfigurable OFDM based transceiver in information security gives high benefits to the wireless communication and used for multipurpose requirements. Reconfigurable transmitters with all the digital modulation techniques are flexible to all the end users without reconfigurable receiver with single modulation technique. Digital 8 bit data which is input to the system that is converted to the high digital signals at the end of receiver side. Receiver output is given to the microcontroller and examining the input data in connected mobile, hence communications between two end users are fully secured.
\end{abstract}

Keywords: Reconfigurable, OFDM, signal to noise ratio (SNR), Multipath Fading, ISI.

\section{Introduction}

During world war it has been very important to communicate between the two countries without spreading their information at that time the term OFDM has evolved by using fully modulated or multiplexing technique it is possible to transmit the digital data over a multicarrier signal with any of the modulation technique. A transmitter is made reconfigurable so that end users have flexibility to choose their own modulation techniques. Growth rate of wireless technology has been accelerated to such a level that it has become ubiquitous by this technique we can easily overcome the problems like Bit error rate, multipath fading, information interchange etc. It was necessary to provide a solution which can offer robustness in multipath environments and against narrowband interference and is efficient. OFDM, in all this aspects, proves to be an apt candidate by not only providing high-capacity, high-speed wireless broadband multimedia networks but also coexists with current and future systems [1]. Orthogonal frequency-division multiplexing (OFDM) is a method of digital modulation in which a signal is split into several narrowband channels at different frequencies. Wireless communication is having the fastest growth phase in history because of unprecedented evolution in the field.

\section{Need For OFDM:}

Single Carrier Modulation System: A typical single carrier modulation spectrum is shown in Figure 1.

$$
\text { I }
$$

$$
\text { Signal Bandwidth }
$$

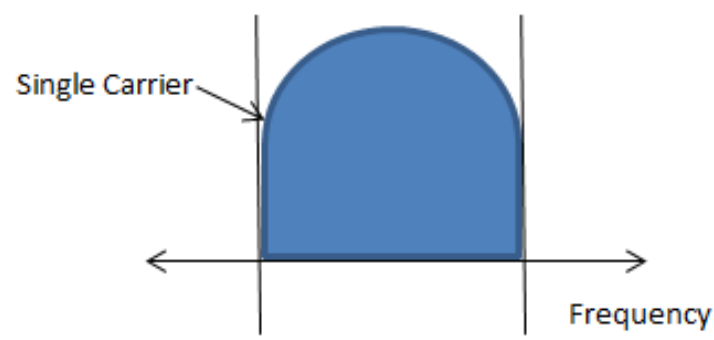

Figure 1: Single carrier Modulation System

A single carrier Modulation System modulated information onto single carrier using variation in amplitude, frequency or phase. Since data rates of current systems tend to be very high, the duration of one bit or symbol (group of bits) becomes very small increasing the bandwidth [2]. The system becomes more susceptible to loss of information through impulse noise, signal reflection, frequency interference and other impairments. The major disadvantage of single carrier system is the inefficient use of bandwidth. 

Transform.

OFDM system is implemented using Fast Fourier Transform and its counterpart Inverse Fast Fourier

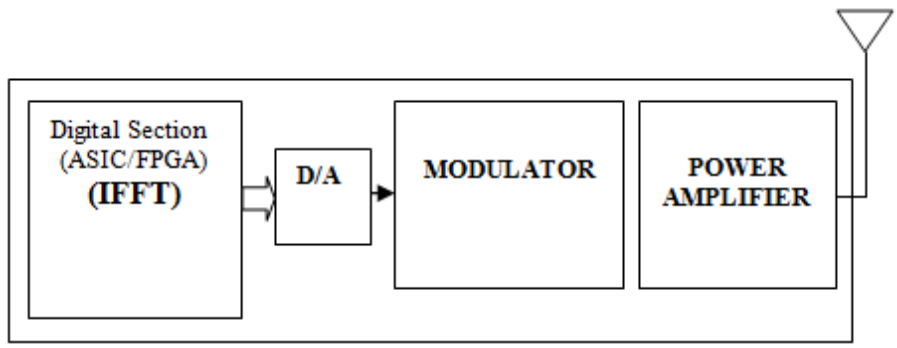

Figure 2: OFDM Transmitter Section

It is mathematically equivalent to DFT and its counterpart IDFT. FFT treats the input as time domain component and converts it into frequency domain component whereas its counterpart IFFT treats the input as frequency domain component and converts it into time domain component. The OFDM transmitter is shown in Figure. 2.

IFFT is used at transmitter section since it consists of orthogonal basis sinusoid function; it treats the input symbol as though they are in frequency domain. These symbols are then converted into time.IFFT takes $\mathrm{N}$ no of symbols where $\mathrm{N}$ is the no of subcarriers in the system. Each symbol has a symbol period which is then mapped onto basis orthogonal sinusoid of IFFT block. These sinusoids have different frequency and the lowest frequency is DC. These input symbols act as a complex weight for orthogonal basis sinusoid function. The amplitude of the complex symbol modulates either the amplitude or phase of the orthogonal subcarrier depending upon the type of modulation used. The output of IFFT is the summation of all $\mathrm{N}$ sinusoids. The block of $\mathrm{N}$ output samples of IFFT make up a single OFDM symbol. The OFDM symbol length is NT where $\mathrm{T}$ is the IFFT input symbol period. The time domain signal from the output of IFFT as shown in Figure is transmitted through channel.

OFDM Receiver:

At the receiver side the signal is amplified, demodulated and then converted to digital form but the signal is in mixed form which is then given to FFT block which then converts the input signal into frequency domain. The output of the FFT is the original symbol transmitted by the transmitter [2].

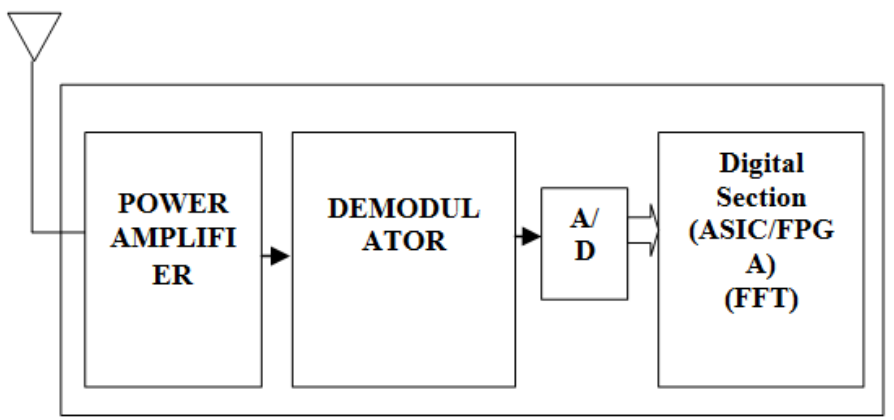

Figure 3: OFDM Receiver Section

The output of the FFT is the original symbol transmitted by the transmitter. It maps the incoming signal into the known basis sinusoid of converts the input signal into frequency domain. The output of the FFT is the original symbol transmitted by the transmitter. It maps the incoming signal into the known basis sinusoid of transmitter and since the signals have orthogonal property it can be recovered with less error at the receiver through Fast Fourier Transform in Figure. 3.

\section{Related Work}

Author [3], in this paper presents the theoretical backgrounds of a QPSK Modulation. The QPSK Modulator is then simulated using Matlab/Simulink environment and System Generator, a tool from Xilinx used for FPGA design as well as implemented on a Spartan 3E Starter Kit board. The modulator algorithm has been implemented on FPGA using the VHDL language on Xilinx ISE 12.3. The local clock oscillator of the board is $50 \mathrm{MHz}$ which corresponds with a period of $20 \mathrm{~ns}$. The frequency of the QPSK carrier is $31,250 \mathrm{kHz}$ and because the QPSK symbol is made of two bits, the output frequency is $62,50 \mathrm{kbps}$. The modulator has been designed and simulated and its performances were evaluated by measurements. 
Author [4], in this paper presents the design of a QPSK digital communication system and its implementation on FPGAs. The simulations of the system were made in Matlab/Simulink environment and System Generator, a tool used for FPGA designs. The system is made up of two Spartan 3E Starter Kit boards. The first is implemented to comport as a modulator and the second as a demodulator. The VHDL hardware description language was used to design each model. The local clock oscillator on the board operates at $50 \mathrm{MHz}$ and used at $31,250 \mathrm{kHz}$ for the carrier. The operating results were compared with the theoretical ones and were also evaluated by measurements.

In this study, author [5], presents the implementation of binary amplitude shift keying (BASK), binary phase shift keying (BPSK), binary frequency shift keying (BFSK), quadrature phase shift keying(QPSK) on FPGA using one of hardware definition language, VHDL. Algorithms are implemented on 3s 100evq 100- 4 device, belong to Xilinx FPGA family. Inputs are from RAM of FPGA card. In this work, the aim of choosing FPGA is: FPGAs perform foster compared to other processors, and parallel-processing features of VHDL enables fast results, to be able to investigate the logical programming side of FPGAs.

In this paper author [6], presents the comparison performance in terms of error performance between two modulation techniques, the BPSK and QPSK modulation. Both modulations were implemented on the Spartan 3E Starter Kit board. In order to compare the error performance of the two modulation techniques, it is necessary to express the error performance in terms of the average energy per bit $(\mathrm{Eb})$. A brief description of theoretical aspects of the BPSK and QPSK modulations is also illustrated in the paper.

Author [7], presents Low-cost and high-performance underwater sensor nodes are important to the dense underwater wireless sensor networks. And the low-cost acoustic communication module is a necessary part for the underwater wireless sensor node. In this paper, a QPSK demodulation method by window mask is proposed. And an underwater acoustic module based on FPGA and QPSK is designed. The acoustic communication module is designed on the software platform QUARTUS II released by ALTERA Corporation. Then the acoustic communication module is realized on Cyclone II chip. Simulations and experiments show that the proposed demodulation method is valid. The developed communication module can process the signal's modulation and demodulation.

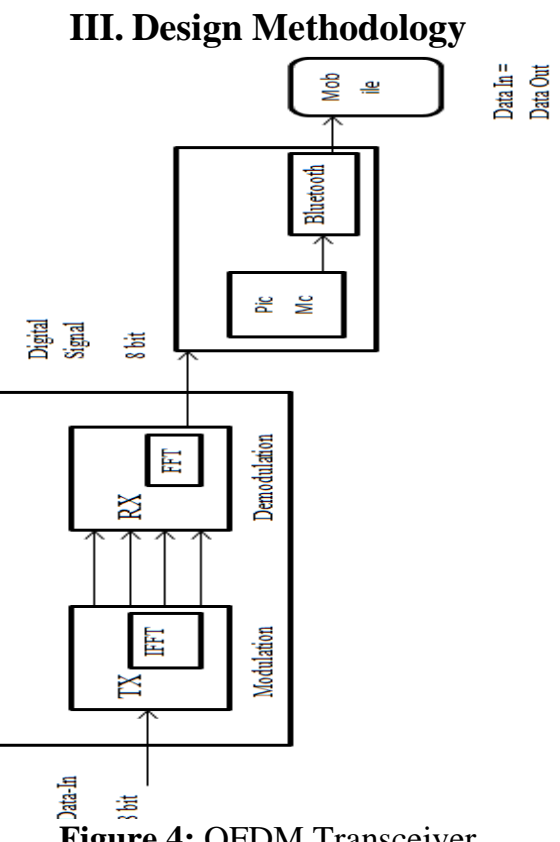

\subsection{Design Of Modulation}

Figure 4: OFDM Transceiver

In digital communications, the modulation process corresponds to switching or keying the amplitude, frequency, or phase of the carrier in accordance with the incoming digital data so there are THREE basic digital modulation techniques are as follows:

1. Amplitude-shift keying (ASK)

2. Frequency-shift keying (FSK)

3. Phase-shift keying (PSK)

- Binary PSK (BPSK)

- Differential PSK (DPSK)

- Quadrature Phase Shift Keying (QPSK)

- $\pi / 4$ Quadrature Phase Shift Keying (QPSK) 


\subsection{Input Sampler}

Any of the modulation or demodulation techniques contain input sampler for each bit sampling. Sampler is also called as 'Bit separator or Bit Splitter' Separating indusial Bits given digital data by Parallel to Serial Conversion Normally called Bit Splitter. This block produces separate bits with its corresponding clock distribution from given input bit stream with respect to clock bit select line. For QPSK Generally input sampler will group the two bits coming from the input section. This section forming two bits called as the one symbol. The output of the input sampler is no. of symbols. This block samples the serial input and generates 2 bit IQ output. The output of the input sampler is group of symbols. Each symbol contains group of two bits.

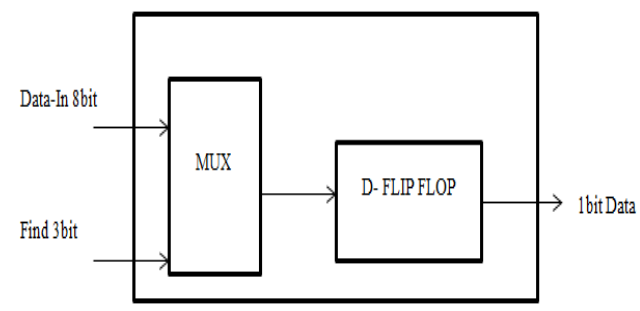

Figure 5: Function Block of Input Sampler

\subsection{Inverse Fast Fourier Transform (IFFT)}

It is the one of the important module in the OFDM system. To compute IFFT first exchange real and imaginary parts then perform FFT. After performing FFT then exchange real and imaginary terms then it is the IFFT and finally divide the by $\mathrm{N}$, where $\mathrm{N}$ is the number of points, here we are using 8 - point FFT so divide the results by 8.The output of the IFFT is applied to the Digital to analog converter is used to convert the digital data into OFDM signals and these symbols are transmitted through the transmitting system.

\subsection{Working}

Working of OFDM based trans receiver is explained as follows the 8 bit binary data is given to the transmitter it uses the any of the modulation technique as present in the receiver side and convert that to digital signal with bit execution rate of $1060 \mathrm{~b} / \mathrm{s}$, by using bit sampler each bit is sampled and given to the analog to digital converter at transmitter side .the out of the transmitter is given to the receiver and again demodulation is done on signal hence output at the receiver is 8 bit digital signal. This transceiver is given to the pic microcontroller of 40 pins that is 32 bit which is intern connected with the Bluetooth device which is paired by cell phone to see and examine the input data given at the transmitter side hence wireless communication is achieved. The VHDL code is written for all the modulation techniques and executed in Xilinx 12.9 environment by using ultra cyclone 4 FPGA, and simulation is done on modalism. Hence following are the results and simulation outputs.

\section{Simulation Results}

A Stimulation is a tool which is used for calculation of different stimulus of different inputs or change in input. Stimulation is done before hardware implementation by doing this we can avoid the unexpected behaviour at any point change in the inputs to get desired output specifications.

Following figure 6. shows the stimulation result for amplitude shift keying (ASK) Hear input for select line is predefined as 000 in the VHDL code and the 8 bit data input given as 11001100 and clock is set and select is set to high that is 1 . Hence the output is at every logic high bit signal appearing and at logic zero there is no signal. Output digital signal is observed at data out and transmitter out is an analog signal of same modulation technique at transmitter out.

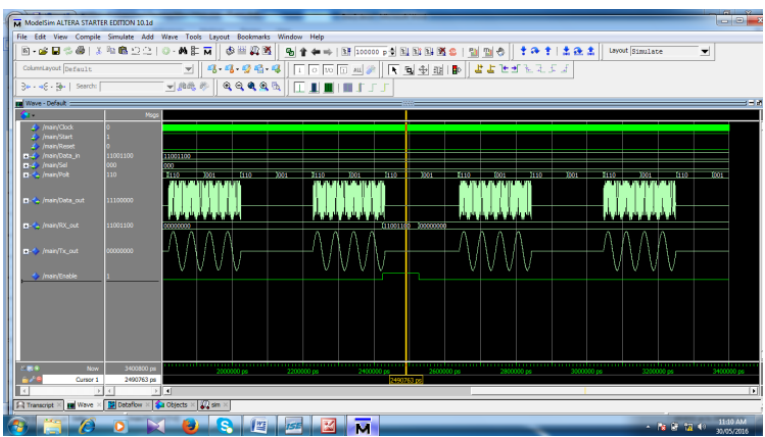

Figure 6: shows the Simulation results of ASK modulation 
Figure 7. shows the FSK output at select value 001 , here signals are frequency shifted at every logic zero, and at logic one signal appears same and increase in time period of the cycle.

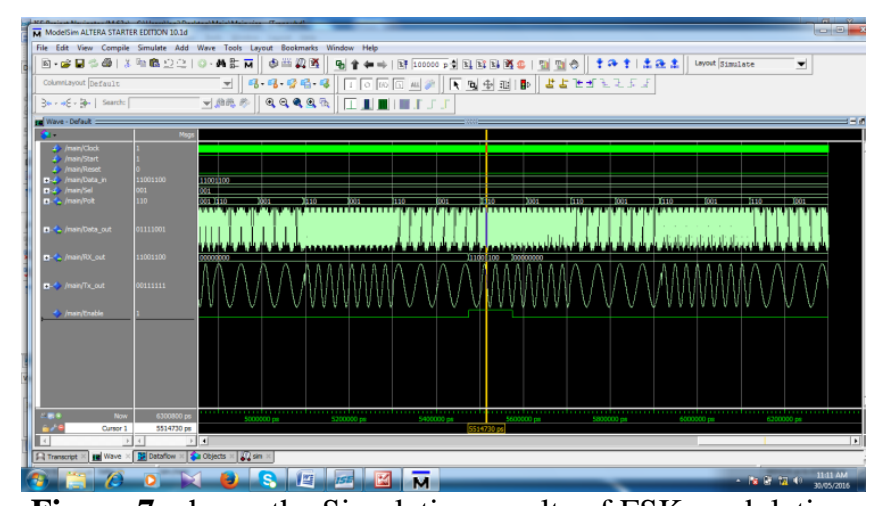

Figure 7: shows the Simulation results of FSK modulation

Figure 8. shows the phase shift keying for QPSK modulation technique at select predefined value of 100. In Quadrature phase shift keying phase is shifted at every logic high or logic low from phase 1 to phase 0 or vice versa.

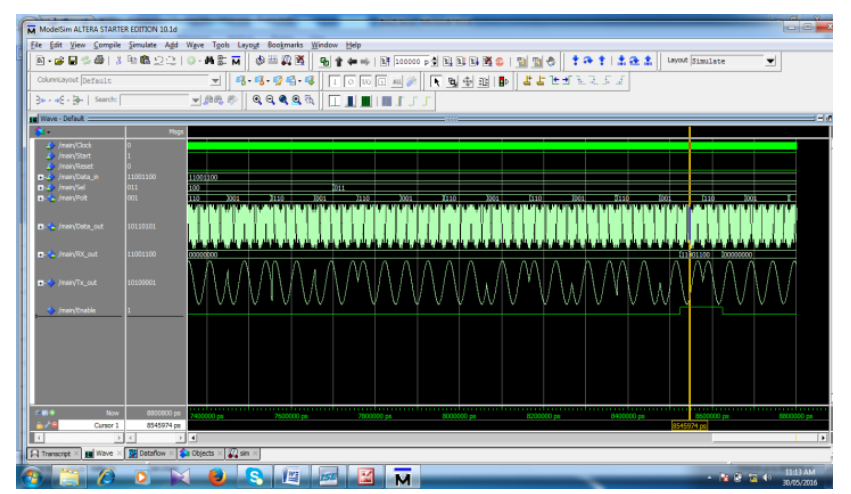

Fig 8: shows the stimulation result of $\pi / 4$ QPSK Modulation

Figure 9. shows the stimulation result for DPSK modulation technique for predefined select value that is 011.these select values are user define can be changed as user convenient in the VHDL code.

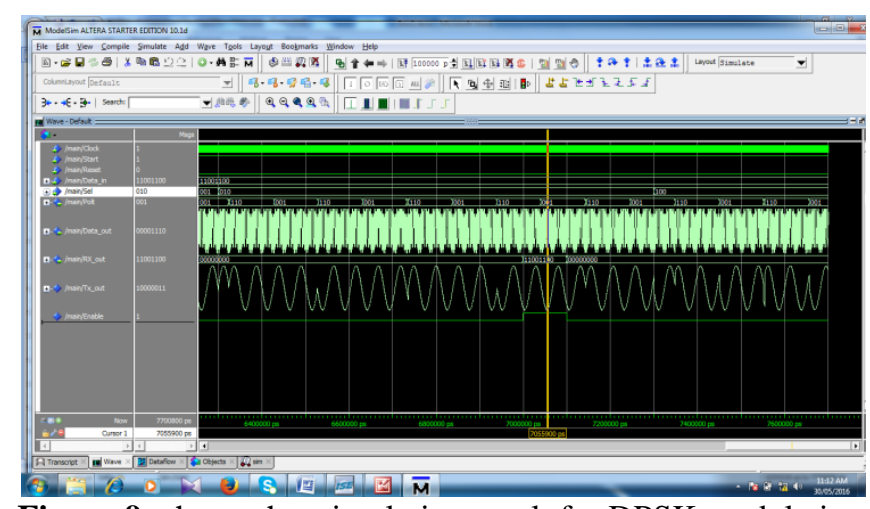

Figure 9: shows the stimulation result for DPSK modulation

Hence all the modulation techniques can be achieved by predefined select line which is defined in the VHDL code. Example: 000-ASK, 001-FSK, 010-PSK, 011-DPSK, 100-PIQPSK.Theses selective bits can be changed as per user convenient in the code.

\section{Conclusion}

This paper mainly deals with the reconfigurable OFDM modulator system which plays an important role in wireless communication. Implementation is done on FPGA and VHDL Code is written for different modulation techniques. Hence hardware functions of OFDM reconfigurable module is done on software and 
which reduces execution cycle time that makes the system response faster in real-time. This leads to a better performance and accuracy. For this phase we have successfully designed all functional modules of modulations like ASK, FSK, BPSK, and QPSK is successfully code in VHDL, simulated in ModelSim all the functions blocks working perfectly hence results are verified.

\section{Reference}

[1]. Beena R. Ballall, Ankit Chadha, "Orthogonal Frequency Division Multiplexing and its Applications", International Journal of Science and Research (IJSR), Volume 2 Issue 1, January 2013, pp 325-328.

[2]. David Gustafsson, Christer M Andersson, Christian Fager,“ A novel wideband and reconfigurable high average efficiency power amplifier," Microwave Symposium Digest (MTT), 2012 IEEE MTT-S International, Volume 1, pp. 1-3.

[3]. Wenmiao Song, "Design and implement of QPSK modem based on FPGA", ICCSIT IEEE, Volume 9, 9-11 July 2010, pp. 599601.

[4]. W.Song, J.Zhang, Q.Yao, "Design and Implementation of BPSK Modulator and Demodulator on Modern DSP Technology", 3rd IEEE International Symposium on Microwave, Antenna, Propagation and EMC Technologies for Wireless Communications, China, 2009, pp.1135-1137.

[5]. G. Elamary, G. Chester, J. Neasham, "A Simple Digital VHDL QPSK Modulator Designed Using CPLD/ FPGAs for Biomedical Devices Applications", in Proceedings of the World Congress on Engineering, Volume. I, 2009, United Kingdom.

[6]. Y. Tachwali, H. Refai, "Implementation of a BPSK Transceiver on Hybrid Software Defined Radio Platforms", 3rd International Conference on Information and Communication Technologies: From Theory to Applications, Syria, 2008, pp. 1-5.

[7]. P.Dondon, J.M.Micouleau, J.Legall, .K.Kadionik, "Design of a low cost BPSK modulator/demodulator for a practical teaching of digital modulation techniques", in the 4th WSEAS/IASME International Conference on Engineering Education, Greece, 2007, pp.61-66.

[8]. Ganiga Raghavendra Chandra, Yadav Santosh Ramroop, "OFDM AND IT’S APPLICATION IN 4G”, 